\section{Inferior Colliculi}

John E. Mendoza

Department of Psychiatry and Neuroscience, Tulane Medical School and SE Louisiana

Veterans Healthcare System, New Orleans, LA, USA

\section{Synonyms}

Nuclei of the inferior colliculi

\section{Definition}

Surface feature of the caudal-most pair of nuclear masses on the posterior surface (tectum) of the midbrain (mesencephalon).

\section{Current Knowledge}

Together with the superior colliculi, which lie directly above, the inferior colliculi make up the corpora quadrigemina. The nuclei of the inferior colliculi are primarily concerned with auditory information. They receive extensive input from the lateral lemniscus, which carries auditory fibers from the superior olivary nuclei and the cochlear nuclei. Both of these latter nuclei receive direct input from the auditory system. In addition to sending fibers to the medial geniculates (the auditory integration and relay nuclei of the thalamus) via the inferior brachium of the inferior colliculus, each nucleus of the inferior colliculus has connections with the nucleus on the opposite side and to the superior colliculi. The roles of the inferior colliculi are not fully understood, but they are thought to be important in the localization of sound and in orienting responses, such as reflexively turning the eyes, head, and neck toward an unexpected or loud sound. The latter is likely accomplished by means of connections with the superior colliculi, which are, in turn, connected to the cervical cord via the tectospinal tract, innervating the muscles of the head and neck. Unilateral lesions of the nucleus of the inferior colliculus are not typically associated with observable deficits, with the possible exception of difficulties with sound localization.

\section{Cross-References}

Auditory System 